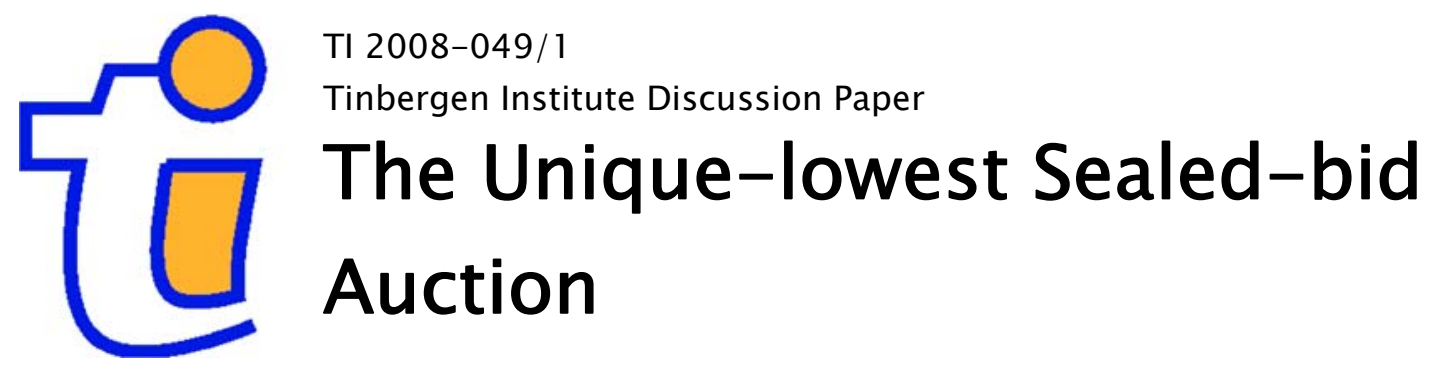

Harold Houba

Dinard van der Laan

Dirk Veldhuizen

VU University and Tinbergen Institute. 


\section{Tinbergen Institute}

The Tinbergen Institute is the institute for economic research of the Erasmus Universiteit Rotterdam, Universiteit van Amsterdam, and Vrije Universiteit Amsterdam.

Tinbergen Institute Amsterdam

Roetersstraat 31

1018 WB Amsterdam

The Netherlands

Tel.: +31(0)205513500

Fax: $+31(0) 205513555$

Tinbergen Institute Rotterdam

Burg. Oudlaan 50

3062 PA Rotterdam

The Netherlands

Tel.: + $31(0) 104088900$

Fax: $+31(0) 104089031$

Most TI discussion papers can be downloaded at http://www.tinbergen.nl. 


\title{
The unique-lowest sealed-bid auction*
}

\author{
Harold Houba ${ }^{\dagger}$ \\ VU University Amsterdam \\ and Tinbergen Institute
}

\author{
Dinard van der Laan \\ VU University Amsterdam \\ and Tinbergen Institute
}

\author{
Dirk Veldhuizen \\ VU University Amsterdam
}

May, 2008

\begin{abstract}
Unique-lowest sealed-bid auctions are auctions in which participation is endogenous and the winning bid is the lowest bid among all unique bids. Such auctions admit very many Nash equilibria (NEs) in pure and mixed strategies. The two-bidders' auction is similar to the Hawk-Dove game, which motivates to study symmetric NEs: Properties and comparative statics are derived and the symmetric NE with the lowest expected gains is the maximin in symmetric strategies, which allows computation through a mathematical program. Simulations provide numerical evidence that the symmetric $\mathrm{NE}$ with the lowest expected gains is the unique limit point of the replicator dynamics.
\end{abstract}

JEL Classification: D44 Auctions, C72 Noncooperative Games, C73 Evolutionary games , C61 Programming Models and Dynamic Analysis, L83 Gambling

Keywords: Sealed-Bid Auction, Evolutionary Stability, Endogenous Entry, Maximin

\footnotetext{
*We are grateful to Quan Wen and Gerard van der Laan for valuable suggestions.

${ }^{\dagger}$ Department of Econometrics, VU University Amsterdam, De Boelelaan 1105, 1081 HV Amsterdam, Netherlands. Email: hhouba@feweb.vu.nl.

${ }_{\ddagger}^{\ddagger}$ Department of Econometrics, VU University Amsterdam, De Boelelaan 1105, 1081 HV Amsterdam, Netherlands.
} 


\section{Introduction}

During the last decade, the new auction format of the lowest-unique-bid auction appeared in several countries. ${ }^{1}$ This format is typically organized by media such as TV, radio and internet. Unlike other types of auctions, this auction does not to seek to generate maximal bids, rather it seeks to maximize bidders' participation from a large 'audience'. Participation is increased by declaring as winner the lowest bid among the unique bids made, being those bids that are made only once. ${ }^{2}$ The items auctioned are new high-priced consumer electronics, cars or monetary prizes. Bids are typically made by sending a costly SMS-call to the auctioneer with the bid expressed in euro cents. Most of the time, the winning bid pays almost nothing. In order to make money, the auctioneer needs to generate revenue from a sufficiently large number of bids, the producer's willingness to pay for having the product on display in the auction and, in case of broadcasting as a TV or radio show, the advertisement revenues from commercial blocks. In some cases, this auction format is used as a marketing instrument meaning the producer is willing to bear losses.

Lowest-unique-bid auctions form a new, rich and nontrivial auction format that is not yet captured by mainstream auction theory, as e.g. surveyed in Krishna (2002). Since such auctions are already run in real life, there is an interest in better understanding this auction format, which is the research topic in Eichberger and Vinogradov (2008), Östling et al. (2007), Rapoport et al. (2007) and this paper. The main focus of these references is to confront theoretical predictions with empirical evidence from either field or laboratory experiments. In contrast, our main focus is a game theoretic analysis in which we focus on two essential issues of these auctions: endogenous costly entry induces uncertainty of how many bids will be made, and how to bid in this auction. For reasons of exposition, we first discuss the main results of our approach and, next, relate these to the references.

\footnotetext{
${ }^{1}$ These countries are Germany, Netherlands, the UK and the US, see Eichberger and Vinogradov (2008), Östling et al. (2007), Rapoport et al. (2007) and Veldhuizen (2006).

${ }^{2}$ Raviv and Virag (2007) report a closely related auction format that ran in Sweden in which bids are restricted to be below 5 or 10 percent of the monetary value of the prize and the highest unique bid wins the auction. This format is also studied in Rapoport et al. (2007).
} 
In this paper, we study a sealed-bid version of the lowest-unique-bid auction in which bidders make a single bid, being a discrete number. Endogenous entry is modelled by allowing the costless bid "do not participate". Since the monetary costs of making a bid are only incurred when the bid (through say SMS) is actually made, this model offers a natural framework to analyze simultaneous entry and bid behavior. Under symmetric strategies, the expected number of bidders follows a binomial distribution with the complementary probability of nonparticipation as the endogenous probability that individual bidders enter. If the probability of nonparticipation is (close to) zero, each bidder enters with probability of (almost) one meaning the variance in the number of participating bidders is (almost) zero. This last property of our model is fundamentally different to the large variance in e.g. large Poisson games, see Myerson (1998) and Myerson (2000), where the variance can only be close to zero if and only if the expected number of participants is close to zero. ${ }^{3}$

Numerical investigation of Nash equilibria in the lowest-unique-bid auction, reveals a large number of asymmetric Nash equilibria (NEs) and this number rapidly increases as value of the monetary prize or the number of bidders increases. This makes a full characterization of NEs impossible. For the parameter values investigated, the lowest-unique-bid auction also admits a unique symmetric NE, to which we turn our main focus. We show that the symmetric NE is always in mixed strategies, always contains the lowest bid in its support, and its support on the subset of numerical bids is always a set of consecutive bids that is typically smaller than the full support on such bids. Moreover, higher bids within the support are played with a lower probability. This reflects the intuition that one should try to bid low, but if everyone would do so, it is better to avoid the overcrowded lower bids and consider the somewhat larger bids. Since larger bids have more potentially winning lower bids, these are less attractive and this is reflected in the decreasing pattern of probabilities. The nonnegative expected gains in this auction format may be positive, in which case all

\footnotetext{
${ }^{3}$ If the number of players $X$ is Poisson distributed with parameter $N>0$, then $E(X)=N$ and $V A R(X)=N^{2}$. So, arbitrary combinations of $E(X) \gg 0$ and $V A R(X) \neq N^{2}$ cannot be studied in large Poisson games.
} 
bidders participates for sure. A positive probability of nonparticipation, however, implies that these gains are zero. We also report a partial invariance of symmetric NE bidding strategies with respect to bidding costs: If, for bidding costs equal to 0, a bidding strategy is a NE strategy with positive expected gains of say 11.3, then this NE strategy remains a NE strategy under all bidding costs 1 up to 11. For larger bidding costs, there exists a NE with expected gains equal to zero. We also perform comparative statics and find sufficient conditions stating that if either the number of bidders or the costs of making a bid becomes too large, then the probability of nonparticipation becomes positive and the expected gains in the symmetric NE are zero. For a sufficiently large value of the prize or zero bidding costs, there is always a positive expected gain and, hence, full participation. This implies that zero bidding costs form a special case and not the general case.

Although the simple two-bidder case allows a closed-form solution, we cannot report such solution for any larger number of bidders, or a proof of uniqueness of the symmetric NE. Under positive expected NE gains and a technical condition, however, we characterize the symmetric NE with lowest expected NE gains as the minimum of a mathematical program. The interpretation of this symmetric NE is that it is the maximin value within the class of symmetric strategies. So, a bidder's bidding strategy also minimizes what this bidder "gives away" in expected NE gains to the other bidders. Recall that positive expected NE gains include the case of zero bidding costs. In case the mathematical program returns expected gains equal to zero, then the symmetric NE with the lowest expected NE also has this property.

From mid-2005 until late 2006, the unique-lowest-bid auction featured in a daily Dutch TV show called Shop4Nop, where "Nop" means "for free", see e.g. Veldhuizen (2006). This raised the question whether the TV audience would learn to play the symmetric NE over time. In addition, we report that the two-bidder case is similar in interpretation to the Hawk-Dove game and, therefore, possesses an evolutionary stable strategy. Since the model is very complex, we resort to a numerical investigation of asymptotic stable equilibria of the 
replicator dynamics as a proxy for evolutionary stable strategies, see Bukowski and Miekisz (2004). For the parameter values run, we observe convergence of these dynamics to the worst symmetric NE.

Our results are complementary to the results in Eichberger and Vinogradov (2008), Östling et al. (2007) and Rapoport et al. (2007). In these references, the emphasis is on the theory needed to derive theoretical predictions for empirical testing. The complexity of the unique-lowest-bid auction is reflected in the different approaches taken in each of these references, and a common conjecture that the symmetric NE is unique.

Under single bids and no bidding costs, Rapoport et al. (2007) provide an innovative unifying framework for analyzing both lowest-unique-bid auctions and highest-unique-bid auctions, as also studied in Raviv and Virag (2007). For this framework, they design a numerical method for approximating the symmetric NE by embedding the model in a nonstationary Markov chain, which is for zero bidding costs a numerically robust alternative to our mathematical program. They do not report other theoretical results and do not mention the issue of endogenous entry.

Östling et al. (2007) assume single bids, no bidding costs and that the winning bidder does not pay his bid, which turns the auction into a guessing game. Their main approach is to model this guessing game as a Poisson game. ${ }^{4}$ Poisson games are somewhat easier to analyze than a standard game in normal form, but these also require a very rigid assumption about the expected number of participants and its variance. For the Poisson guessing game, Östling et al. (2007) show uniqueness, a full support over all feasible bids, bidding probabilities that decrease as the bids increase, and convergence to the uniform distribution as the number of bidders goes to infinity. Our results indicate that some of these results are also valid in a standard game. There are, however, also subtle differences: The full support result is due the assumption of a guessing game. And, for positive bidding costs, we also have convergence to a limit distribution over numerical bids as the number of bidders goes to

\footnotetext{
${ }^{4}$ They also analyze the guessing game as a classical game and report that numerically solving the equilibrium conditions is explosive in the number of bidders and is better avoided for more than eight bidders.
} 
infinity, albeit a trivial sort of uniform distribution with limit probabilities equal to zero because the individual probability of nonparticipation goes to one. This once more illustrates that endogenous costly entry cannot be ignored in either the lowest-unique-bid auction or its related guessing game.

The extension of our model to multiple bids is analyzed in Eichberger and Vinogradov (2008). In this reference, a closed-form solution is stated that is the symmetric NE for the cases of two-bidders and three-bidders, but they also report a counter example with seven bidders. Generally speaking, their main result states that, for some endogenous consecutive sets of bids that contains the lowest bid, bidders randomize over all subsets (of this endogenous set) that contain the lowest bid. Therefore, single bids do not arise endogenously from the multiple-bids model. This implies that the single bid model requires an analysis on its own, as is done in this paper.

The overall conclusion is that the results in Eichberger and Vinogradov (2008), Östling et al. (2007), Rapoport et al. (2007) and this paper all indicate at similar qualitative insights that are quite robust with respect to a standard game-theoretic versus Poisson-game approach and single versus multiple bids. Our paper enriches the literature by raising the issue of endogenous entry, the maximin interpretation of the symmetric NE and numerical evidence for evolutionary stability.

This paper is organized as follows. We first introduce our model in Section 2. In Section 3, we discuss several motivating examples that identify the main issues of concern. All theoretical results are derived in Section 4 and 5, where the latter section contains the mathematical program. A numerical analysis of the replicator dynamics and the mathematical program are reported in Section 6. This paper concludes with some remarks.

\section{The lowest-unique sealed-bid auction}

The lowest-unique sealed-bid auction is an anonymous auction in which $n+1 \geq 2$ bidders either stay out or enter by making a single sealed bid for some mass-produced consumer 
good. This good is for sale everywhere at (internet) shops for $M \gg 0$ monetary units, say cents. Making a bid costs $0<c \ll M$ monetary units. We assume that $n, M$ and $c$ are common knowledge. The winning bid is the lowest bid among all bids that are unique among the monetary bids made. The winner of the auction pays his own bid. Note that the existence of a winning bid is not guaranteed, as for example $n+1$ identical monetary bids demonstrate. In the absence of a winner, the item remains with the auctioneer.

Formally, bidders are numbered $b=1,2, \ldots, n+1$ and each bidder faces the same set of feasible bids, denoted as $B$. We write $\beta^{b} \in B$ for bidder b's bid. Participation is voluntary, and we capture the option not to participate $(N)$ by including the bid $\beta^{b}=N .^{5}$ All other bids are monetary bids $\beta^{b} \in \mathbb{N}$ that cost $0<c \ll M$ and that are all consecutive multitudes of the monetary unit with zero as the smallest bid. ${ }^{6}$ Since the winner pays his own bid and the item is elsewhere for sale for $M$, bidding strictly more than $M-c$ is irrational. Therefore, it is without loss of generality to assume $B=\{N\} \cup\{0,1, \ldots, M-c\}$. For its subset of monetary bids we write $B^{N}=B \cap \mathbb{N}$. The simplex of mixed strategies on $B$ is denoted as $\Delta=\left\{x \in \mathbb{R}_{+}^{M-c+2} \mid \sum_{i \in B} x_{i}=1\right\}$ and we write $x^{b} \in \Delta$ for bidder $b$ 's mixed strategy.

An outcome of the auction is denoted as $\beta=\left(\beta^{b}, \beta^{-b}\right) \in B^{n+1}$. Every outcome $\beta \in B^{n+1}$ specifies a possibly empty set of unique monetary bids and, if not empty, the winner is the bidder who has the lowest unique bid in this set. Given $\beta \in B^{n+1}$, we define the identity of the winning bidder as $I(\beta) \in\{\{1\}, \ldots,\{n+1\}\} \cup \emptyset$ with the understanding that $I(\beta)=\emptyset$ indicates no winner. Bidder $b$ 's risk neutral utility function $u_{b}: B^{n+1} \rightarrow \mathbb{R}$ is defined as

$$
u_{b}\left(\beta^{b}, \beta^{-b}\right)= \begin{cases}0, & \text { if } \beta^{b}=N, \\ M-c-\beta^{b}, & \text { if } I\left(\beta^{b}, \beta^{-b}\right)=b, \\ -c, & \text { otherwise. }\end{cases}
$$

This completes the description of the lowest-unique sealed-bid auction as a game in normal form with complete information and finite, discrete pure strategy sets. Hence, the standard

\footnotetext{
${ }^{5}$ The normal-form game specified can be seen as the reduced form of a two-stage game with unobservable entry in which bidders first decide whether to participate $(P)$ and, if so, then decide on the sealed bid. In such extensive form, a bidder's strategy specifies two pairs of the form $(P, \beta)$ and $(N, \beta), \beta \in \mathbb{N}$, where all bids of the second form are payoff equivalent making the $P$ in the first form superfluous.

${ }^{6}$ Our setup captures all cases in which the rules specify a minimum bid of $b^{\text {min }}>0$, because then an item worth $M-b^{\text {min }}$ normalizes the auction to one with a lowest bid of zero.
} 
existence result for a mixed-strategy Nash equilibrium (NE) applies.

We conclude this section by characterizing the minimax value as the nonparticipation payoff by identifying a NE strategy profile that supports it.

Proposition 1 Bidder b's minimax value is 0 and can be supported by a pure NE strategy profile. Moreover, for every NE, the NE payoffs are nonnegative.

Proof. The bid $\beta^{b}=N$ secures a payoff of 0 for bidder $b$. All other bidders can hold bidder $b$ down to 0 by appropriate coordination on $\beta^{-b}$ such that a single bidder $k \neq b$ bids $\beta^{k}=0$ and all others bid $\beta^{l}=N, l \neq b, k$. Hence, 0 is bidder $b$ 's minimax value. It can be verified that these pure minimax strategies describe a Nash equilibrium. For every NE, the NE payoffs weakly dominate the minimax payoffs, which is 0 .

Corollary 2 There exist asymmetric pure NE strategy profiles supporting each bidder's nonparticipation payoff 0 and this bidder's utopia payoff $M-c$.

The asymmetric NE strategy profile in the proof of Proposition 1 that supports the minimax values requires that all bidders coordinate who will be the single bidder bidding zero and obtains the auctioned item against cost of bidding $c$, while all others do not participate. There are always $n+1$ of such asymmetric pure-strategy NE. From the perspective of the auctioneer, the bidding behavior is identical to that of a bidding ring.

\section{Motivating examples}

A formal analysis of the lowest-unique sealed-bid auction would aim at the characterization of the set of NE. In this section, however, we argue that such aim is too ambitious for the auction under consideration. This insight is derived from the following examples.

For $n+1=2$ we have a closed form solution of the set of all NE. In this case, we have the following bi-matrix game: 


\begin{tabular}{|c|c|c|c|c|c|}
\hline & $N$ & 0 & 1 & $\cdots$ & $M-c$ \\
\hline$N$ & 0,0 & $0, M-c$ & $0, M-c-1$ & $\ldots$ & 0,0 \\
\hline 0 & $M-c, 0$ & $-c, \quad-c$ & $M-c, \quad-c$ & $\ldots$ & $M-c,-c$ \\
\hline 1 & $M-c-1,0$ & $-c, M-c$ & $-c$ & $\cdots$ & $M-c-1,-c$ \\
\hline$\vdots$ & $\vdots$ & $\vdots$ & $\vdots$ & $\ddots$ & $\vdots$ \\
\hline$M-c$ & 0,0 & $-c, M-c$ & $-c, M-c-1$ & $\ldots$ & $-c,-c$ \\
\hline
\end{tabular}

The asymmetric pure-strategy NE already described are $(N, 0)$ and $(0, N)$. Furthermore, there is a symmetric mixed-strategy NE in which each bidder bids 0 with probability $1-\frac{c}{M}$ and does not participate with probability $\frac{c}{M} \cdot{ }^{7}$ In the symmetric NE, the probability that nobody participates is $\left(\frac{c}{M}\right)^{2}$ meaning the expected number of bidders increases in $M$ and decreases in $c$. None of the bidders expects to make a profit from participating because expected NE utility is always zero. The probability that nobody wins the auction is $\left(\frac{c}{M}\right)^{2}+$ $\left(1-\frac{c}{M}\right)^{2}$

There is an interesting similarity in interpretation with the Hawk-Dove (HD) game. In the HD game two animals might fight for a single prize but face individual costs of fighting that are larger than the worth of this prize, see e.g. the survey in Weibull (1995). In both games, the "bidders" contest for a valuable object, but contesting is costly. Both games have two asymmetric NE in which only one bidder contests for the object, while the other refrains from doing so. Similar, they both have a symmetric mixed NE. So, the general uniquelowest sealed-bid auction can be seen as a generalized HD game with a single prize, multiple contesters and a richer strategy structure for each contester. The similarity for $n+1=2$ goes even further: If we restrict the auction's strategy space to $\{N, 0\}$, then both this restricted auction and the HD game can be reduced to the same generic form with negative diagonal elements. Hence, the mixed NE is also the unique evolutionary stable equilibrium (ESS) in the restricted auction.

Next, consider the lowest-unique sealed-bid auction with $n+1=3, M=5$ and $c=1$. In order to numerically compute all NE, we applied the software tool GAMBIT, see e.g. McKelvey et al. (2006). Doing so, yields twenty-one asymmetric NE and one symmetric

\footnotetext{
${ }^{7}$ Note that in the limit as $c$ goes to 0 participation would be costless and each bidder bids $\beta^{b}=0$ for sure in the symmetric NE, meaning no winner for sure.
} 


\begin{tabular}{|ccc|cccc|cccc|}
\hline \multicolumn{3}{|c|}{ Bidder 1 } & \multicolumn{5}{c|}{ Bidder 2} & \multicolumn{5}{c|}{ Bidder 3 } \\
\hline$x_{0}$ & $x_{1}$ & $x_{2}$ & $x_{N}$ & $x_{0}$ & $x_{1}$ & $x_{2}$ & $x_{N}$ & $x_{0}$ & $x_{1}$ & $x_{2}$ \\
\hline 1.0000 & & & 1.0000 & & & & 1.0000 & & & \\
& 1.0000 & & .2000 & .8000 & & & .2000 & .8000 & & \\
.7000 & .3000 & & .6667 & .3333 & & & .6667 & .3333 & & \\
.6851 & .3149 & & .6351 & .3649 & & & .5842 & .3649 & .0509 & \\
.6049 & & .3951 & & .4495 & .5505 & & & .4495 & .5505 & \\
.5672 & .4328 & & & .4882 & .2133 & .2986 & & .4882 & .2133 & .2986 \\
\hline
\end{tabular}

Table 1: Asymmetric NE ordered by bidder 1's expected NE payoffs, see Table 3.2.

\begin{tabular}{|cccc|}
\hline \multicolumn{4}{|c|}{ Exp. NE payoff } \\
\hline Bidder 1 & Bidder 2 & Bidder 3 & Sum \\
\hline 4.0000 & 0.0000 & 0.0000 & 4.0000 \\
1.7200 & 0.0000 & 0.0000 & 1.7200 \\
1.2222 & 0.0000 & 0.0000 & 1.2222 \\
1.0166 & 0.0000 & 0.0000 & 1.0166 \\
0.5153 & 0.0876 & 0.0876 & 0.6905 \\
0.3098 & 0.1076 & 0.1076 & 0.5250 \\
\hline
\end{tabular}

Table 2: Asymmetric NE utilities of Table 1 ordered by bidder 1's expected NE payoffs.

NE, which is more than seven times the number of NE found for the two-bidder auction. Of these asymmetric NE, many are essentially the same in the sense that these only differ with respect to the permutation of the bidders' roles. Omitting such permutations, these twenty-one reduce to the six cases reported in Table 1 and 2 that are ordered with respect to bidder 1's expected NE payoffs. In all asymmetric NE, bidder 1 enters for sure and for that reason we omitted $x_{N}^{1}$ in Table 1 . Five of these six asymmetric NE feature symmetric strategies for bidders 2 and 3 and each allows for three permutations, which accounts for fifteen asymmetric NE. The fourth asymmetric NE has different roles for each bidder and has six permutations of the bidders' roles.

These asymmetric NE feature a variety of equilibrium behavior. Bidder 1's second-best asymmetric NE features bidder 2 and 3 bidding as if participating in the two-bidder auction while bidder 1 gambles that bidding 1 wins, which has probability $\left(\frac{1}{5}\right)^{2}+\left(\frac{4}{5}\right)^{2}=\frac{17}{25}$. Bidder 1's third-best asymmetric NE features similar bidding behavior by bidder 2 and 3 but they 
participate by bidding 0 with the lower probability of $\frac{1}{3}$, while bidder 1 spreads his bid over the two lowest bids. From the perspective of bidder $1, \beta^{1}=0$ and $\beta^{1}=1$ win with probability $\frac{4}{9}$, respectively, $\frac{4}{9}+\frac{1}{9}$ and both bids have an expected payoff of $\frac{11}{9}$. These two types of NE illustrate that slightly bidding more than the other bidders might be a profitable strategy to avoid the fierce competition for the lowest bid. Bidder 1's fourth-best NE features bidder 1 and 2 spreading their risk over the two lowest bids, albeit that bidder 1 participates for sure while bidder 2 is less bold, and bidder 3 only competes for the lowest bid if he participates. Bidder 1's one but worst NE has all three bidders participating for sure, all compete with positive probability for the lowest bid, and bidder 1 avoids the expected competition for the one but lowest bid between bidder 2 and 3. In bidder 1's worst NE, all bidders enter for sure and bidder 1 has less spread over monetary bids than the other two bidders.

This numerical example also is a counter example in showing that several types of results cannot be obtained for all asymmetric NEs:

1. Bidder 1's second-best NE excludes that the support of monetary bids always includes the lowest bid 0 .

2. Bidder 1's fifth-best NE excludes that the support of monetary bids always consists of consecutive numbers.

3. Bidder 1's second-best NE and third-best NE exclude a negative relation between bidder 1's expected NE payoff and the (sum of) participation probabilities of the other bidders.

4. Bidder 1's expected NE payoffs show no obvious relation with the supports of monetary bids.

As mentioned, there is also a unique symmetric NE. It is in mixed strategies that are not fully mixed over $B$. Comparative statics with respect to the value of $M$ in GAMBIT, as reported in Table 3, shows that $M=5$ is the lowest $M$ for which all bidders participate 


\begin{tabular}{|r|ccccc|r|r|}
\hline$M$ & $x_{N}$ & $x_{0}$ & $x_{1}$ & $x_{2}$ & $x_{3}$ & NE payoff & Sum payoffs \\
\hline 3 & .5669 & .4227 & .0105 & & & 0.0000 & 0.0000 \\
4 & .2887 & .5000 & .2113 & & & 0.0000 & 0.0000 \\
5 & & .5191 & .3407 & .1403 & & 0.1565 & 0.4695 \\
6 & & .5093 & .3189 & .1717 & & 0.4447 & 1.3342 \\
10 & & .4904 & .2902 & .2154 & .0040 & 1.5969 & 4.7907 \\
15 & & .4801 & .2769 & .1750 & .0680 & 3.0544 & 9.1632 \\
25 & & .4713 & .2659 & .1577 & .1051 & $* * *$ & $* * *$ \\
\hline
\end{tabular}

Table 3: Symmetric NEs for several values of $M, n+1=3$ and $c=1$.

for sure because the expected NE payoffs are positive. For smaller $M$, nonparticipation has positive probability in the symmetric NE and the bidders' expected NE payoffs are zero. The support of monetary bids consists of decreasing probabilities on consecutive numbers and its size increases in $M$. The symmetric NE under GAMBIT can also be computed as the quantal response equilibrium, see McKelvey and Palfrey (1995). Although this equilibrium concept suggest an evolutionary underpinning, examples exist for which such equilibrium fails to be evolutionary stable.

We also ran other parameter values under $n+1=3$ in GAMBIT. The number of NEs grows very rapidly, for example for $M=15$ and $c=1$, we obtained 43 NEs in total of which only one was symmetric. All these numerical examples are devastating for the prospect of characterizing the set of NEs for arbitrary $n+1, c$ and $M$. In the next sections we investigate symmetric NE for the following reasons. The existence of so many asymmetric NE demands a lot of coordination that goes beyond the correct anticipation on a particular NE, because the coordination also involves each bidder playing the correct role. For people on the internet community we expect $n$ to be large and that they hardly know each other. This suggests that the required coordination on asymmetric NE is very implausible to occur. Since the unique symmetric NE is the only NE that does not require coordination on bidders' roles, this makes symmetry somewhat less demanding. Besides, these internet auctions are run regularly and bidding behavior might evolve over time. The similarity with the HD game mentioned earlier then suggest the stability of the symmetric NE should be part of 
the analysis. We split our analysis into an investigation of the properties and, next, the evolutionary stability of symmetric NEs.

\section{Symmetric Nash Equilibrium}

In this section, we establish several properties of symmetric NEs and, then, perform some comparative statics with respect to the number of bidders. Since we already characterized the class of two-bidder auctions, we assume without loss of generality that $n+1 \geq 3$.

We first establish existence of a symmetric NE in our symmetric model.

Proposition 3 There exists a symmetric NE and every symmetric NE features randomization.

Proof. The conditions of Theorem 1 in Becker and Damianov (2006) hold, which proves the existence of a symmetric mixed-strategy NE. Any symmetric NE must involve the randomization over at least two bids in $B$. Suppose not, then for some $\alpha \in B$ the pure strategy profile $\beta=(\alpha, \ldots, \alpha)$ forms a symmetric NE. In such NE: $I\left(\beta^{b}, \beta^{-b}\right)=\emptyset$ and $b$ 's payoff is at most 0 , if not negative. If $\alpha \neq 0$, then $\beta^{b}=0$ is bidder $b$ 's optimal deviation with $I\left(0, \beta^{-b}\right)=b$ for sure and $u_{b}\left(0, \beta^{-b}\right)=M-c>0$. Similar, for $\alpha=0, \beta^{b}=1$ is the optimal deviation $I\left(1, \beta^{-b}\right)=b$ for sure and $u_{b}\left(1, \beta^{-b}\right)=M-c-1>-c$. Hence, there does not exist any pure symmetric NE.

From here on, we mean by $x \in \Delta$ is a symmetric NE that that $x^{b}=x$ for all $b=1, \ldots, n+1$ is a symmetric mixed strategy NE. The non-empty set of symmetric NE is denoted as $\Delta^{N E}$. The support of $x$ is denoted as $S(x) \subseteq B$ and for its subset of monetary bids we write $S^{N}(x) \subseteq B^{N}$

The numerical examples of Section 3 all had a symmetric NE in which the lowest bid was chosen with a positive probability, the support of numerical bids is a set of consecutive numbers and the probabilities decreases when bids in the support increase. The following theorem states that these properties generally hold in any symmetric NE. 
Theorem 4 Let $x \in \Delta^{N E}$. Then, the support $S^{N}(x)$ is a set of consecutive numbers that contains the lowest bid of 0 . The probability $x_{i}$ is strictly monotone decreasing in $i \in S^{N}(x)$ and the probability of winning the auction with bid $i$ is strictly monotone increasing in $i \in$ $S^{N}(x)$

Before proceeding to the formal proof, we first discuss this result. A support of consecutive numbers means that there are no gaps of bids that will never be chosen. The rationale is that any bid in such gap would allow to undercut all NE bids above it by making a unique bid for sure, and if such bid would prevail as the winning bid, it is also less expensive. Since the probability is positive that such undercutting bid is winning it does therefore strictly better than the NE bids above this gap. This argument also implies that the lowest bid should not be part of any gap and, therefore, must be included in the support.

Symmetric NE underpin the complicated deliberations of bidding in the lowest-unique sealed-bid auction. On the one hand, the rules imply that bidding the lowest bid or close to it should be considered, because otherwise someone else might undercut. On the other hand, if everybody bids very low, the lower bids become overcrowded and it is better to contemplate a somewhat higher bid. Since such bids have more potential lower bids that might be winning, these bids are less attractive. This is reflected in that such bids are made with lower probabilities. The mixed symmetric NE indicates that lower bids are more likely to be observed in such an auction than the higher bids. To make the more costly higher bids more attractive, they offer a higher probability of winning to offset the extra costs and the risk that lower bids prevail as the winning bid.

We now turn our attention to the proof of Theorem 4. For notational convenience, we take the perspective of bidder 1 and, then, define $F_{i} \subseteq B^{n}, i \in B^{N}$, as the set of partial outcomes $\beta^{-1}=\left(\beta^{2}, \ldots, \beta^{n+1}\right)$ such that $I\left(i, \beta^{-1}\right)=1$, meaning $i$ is a winning bid for bidder 1 given $\beta^{-1}$. This implies that, if $k_{j}\left(\beta^{-1}\right)$ is the number of bids equal to $j \in B^{N}$ in $\beta^{-1}$, then $\beta^{-1} \in F_{i}$ if and only if $k_{j}\left(\beta^{-1}\right) \neq 1$ for all $j=1, \ldots, i-1$ and $k_{i}=0$. 
Given the mixed strategy $x \in \Delta$, let $f_{i}(x)$ be the probability that bidder 1 can win by making the bid $i \in B^{N}$ given that all other bidders use the mixed strategy $x$, i.e., $x^{-1}=(x, \ldots, x)$. Then, we have for every $i \in B^{N}$ that

$$
f_{i}(x)=\sum_{s \in F_{i}} \operatorname{Pr}\left(\beta^{-1}=s\right)=\sum_{s \in F_{i}} x_{s^{2}} \cdot \ldots \cdot x_{s^{n+1}} \equiv \sum_{s \in F_{i}} \operatorname{Pr}(s),
$$

where $s=\left(s^{2}, \ldots, s^{n+1}\right) \in B^{n}$. Obviously, $f_{i}(x)$ is continuous in $x$. Bidder 1's expected utility of bid $i \in B^{N}$ against $x^{-1}$ is denoted as $u(i, x)$ and it is equal to $(M-i) f_{i}(x)-c$. The expected symmetric NE utility is denoted as $u(x, x)$ and it is a continuous function in both $x^{1}=x$ and $x^{-1}=(x, \ldots, x)$.

We have the following property for the support of any symmetric NE.

Lemma 5 Let $x \in \Delta^{N E}$. There exists a $\bar{\beta} \in B^{N}$ such that $S^{N}(x)=\{0, \ldots, \bar{\beta}\}$.

Proof. By Proposition $3, S^{N}(x) \neq \emptyset$. Suppose $S^{N}(x)$ is not a set of consecutive numbers, then there exists a number $i \notin S^{N}(x)$ and a partitioning of $S^{N}(x)$ into $L$ (low) and $H$ (high) such that both $L, H \neq \emptyset, L \cup H=S^{N}(x)$ and $i$ is larger than all bids in $L$ but smaller than all bids in $H$. For every $h \in H$ it holds that $f_{h}(x)>0$, because otherwise the expected payoff of bidding $h$ would be $-c<0$, a contradiction. If $h \in H$ turns out to be the winning bid, then so would have done the bid $i<h$.

Consider the deviation $x^{\prime} \in \Delta$ such that $x_{j}^{\prime}=x_{j} \geq 0$ for $j \in\{N, 0, \ldots, i-1\}, x_{i}^{\prime}=$ $\sum_{h \in H} x_{h}>0$, and $x_{j}^{\prime}=0$ for all $j \geq i+1$. Then, i) $i$ is the winning bid in all cases where some $h \in H$ would have been the winning bid, ii) the event " $i$ wins" occurs with positive probability under $x^{-1}$ and iii) $i<h$ for all $h \in H$ implies bidder $b$ pays less if he wins. But then, the deviating strategy $x^{\prime}$ has a higher expected utility than $x \in \Delta^{N E}$, a contradiction. Hence, the support $S^{N}(x)$ of $x \in \Delta^{N E}$ is a set of consecutive numbers.

Finally, we cannot have $\min S^{N}(x)>0$, because then the previous argument applies for $i=0, L=\emptyset$ and $H=S^{N}(x)$. Hence, $\min S^{N}(x)=0$.

The following lemma follows directly from the definition of the NE concept: All pure strategies in the support of a mixed NE are optimal pure strategies and payoff equivalent. 
Lemma 6 Let $x \in \Delta^{N E}$. For all $i \in S^{N}(x)$ and $j \in B^{N},(M-i) f_{i}(x) \geq(M-j) f_{j}(x)$ and equality holds if also $j \in S^{N}(x)$. Moreover, $N \in S(x)$ implies $u(x, x)=0$.

Lemma 6 implies the following result, which completes the proof of Theorem 4.

Lemma 7 If $x \in \Delta^{N E}$, then $x_{i}$ and $f_{i}(x)$ are strictly monotone decreasing, respectively, increasing in $i \in S^{N}(x)$.

Proof. Suppose that for $i, i+1 \in S^{N}(x)$ we would have $x_{i+1} \geq x_{i}$. Let $\beta^{-1} \in F_{i+1}$. Then for $j=0, \ldots, i$ we have that $k_{j}(x) \neq 1$ and $k_{i+1}=0$. From $\beta^{1}$ we construct bids $\hat{\beta}^{-1}=\hat{\beta}^{-1}\left(\beta^{-1}\right)$ such that $\hat{\beta}^{-1} \in F_{i}$ by moving all bids $\beta^{b}=i, b=2, \ldots, n+1$, to $\hat{\beta}^{b}=i+1$. Formally, let $\hat{\beta}^{-1}=\hat{\beta}^{-1}\left(\beta^{-1}\right)=\left(\hat{\beta}^{2}, \ldots, \hat{\beta}^{n+1}\right)$, where for $b=1, \ldots, n$ we take $\hat{\beta}^{b}=i+1$ if $\beta^{b}=i$ and $\hat{\beta}=\beta^{b}$ otherwise. Then, for $\hat{\beta}^{-1}$ we have that $k_{j} \neq 1$ for $j=0,1, \ldots, i-1$ and $k_{i}=0$. Hence, $\hat{\beta}^{-1} \in F_{i}$. Moreover, from $x_{i+1} \geq x_{i}$ it follows that $\operatorname{Pr}\left(\hat{\beta}^{-1}\left(\beta^{-1}\right)\right) \geq \operatorname{Pr}\left(\beta^{-1}\right)$, because all $x_{s^{b}}=x_{i}$ become $x_{s^{b}}=x_{i+1}$ in (1). But then we have

$$
f_{i+1}(x)=\sum_{\beta^{-1} \in F_{i+1}} \operatorname{Pr}\left(\beta^{-1}\right) \leq \sum_{\hat{\beta}^{-1}\left(\beta^{-1}\right) ; \beta^{-1} \in F_{i+1}} \operatorname{Pr}\left(\hat{\beta}^{-1}\left(\beta^{-1}\right)\right) \leq \sum_{s \in F_{i}} \operatorname{Pr}(s)=f_{i}(x)
$$

and this implies $(M-(i+1)) f_{i+1}(x)<(M-i) f_{i}(x)$ with $i, i+1 \in S^{N}(x)$. This contradicts Lemma 6. Thus, if $i, i+1 \in S^{N}$ then $x_{i+1}<x_{i}$. Finally, by Lemma 6 , for any $i, i+1 \in S^{N}(x)$, we have that $f_{i}(x)=\frac{M-i-1}{M-i} f_{i+1}(x)<f_{i+1}(x)$.

The probability $f_{i}(x)$ of bid $i$ being the winning bid can also be expressed by the following formulas:

$$
\begin{aligned}
f_{0}(x) & =\left(1-x_{0}\right)^{n}, \\
f_{1}(x) & =\sum_{k_{0} \neq 1}^{n}\left(\begin{array}{c}
n \\
k_{0}
\end{array}\right) x_{0}^{k_{0}}\left(1-x_{0}-x_{1}\right)^{n-k_{0}}, \\
f_{i}(x) & =\sum_{\substack{k_{0}+k_{1}+\ldots+k_{i-1}+k_{i}=n \\
k_{j} \neq 1, j=0,1, \ldots, i-1}}^{n}\left(\begin{array}{c}
n \\
k_{0}, k_{1}, \ldots, k_{i-1}, k_{i}
\end{array}\right) \cdot x_{0}^{k_{0}} \cdot x_{1}^{k_{1}} \cdot \ldots \cdot x_{i-1}^{k_{i-1}} \cdot r_{i}^{k_{i}}(x),
\end{aligned}
$$


where $r_{i}(x)=1-x_{0}-\ldots-x_{i}$ is the complementary probability that an individual bid does not belong to the set $\{0,1, \ldots, i\}$ and the multinomial coefficient is defined as

$$
\left(\begin{array}{c}
n \\
k_{0}, k_{1}, \ldots, k_{i-1}, k_{i}
\end{array}\right)=\frac{n !}{k_{0} ! \cdot k_{1} ! \cdot \ldots \cdot k_{i-1} ! \cdot k_{i} !} .
$$

From $f_{0}(x)=\left(1-x_{0}\right)^{n}$ and $u(x, x)=u(0, x) \geq 0$, we can derive

$$
x_{0}=1-\left(\frac{c+u(x, x)}{M}\right)^{\frac{1}{n}} \leq 1-\left(\frac{c}{M}\right)^{\frac{1}{n}} .
$$

However, except for the quadratic case $n+1=3$, the powers in these probabilities for $i \geq 1$ become too large in order to obtain similar closed-form solutions for $x_{1}, x_{2}, \ldots$ or to characterize all NEs. So, one has to resort to numerical analyses in studying symmetric NEs or comparative statics.

We conclude this section with a comparative static result for large numbers of bidders, large costs of making a bid and large value of the prize. In order to state this result, we need to define $\Delta^{N E}(M, c, n+1)$ as the set of symmetric NE for $M, c$ and $n+1$.

Theorem 8 If the number of bidders is sufficiently large $\left(n+1 \geq \frac{M}{c}\right)$ or the cost of making a bid are sufficiently large $\left(c \geq \max _{x \in \Delta^{N E}(M, 1, n+1)} f_{0}(x) M\right)$, then in any symmetric $N E$ there is a positive probability of nonparticipation and the expected NE payoffs are zero. In particular, as the number of bidders goes to infinity, the probability of nonparticipation goes to one. For sufficiently large monetary value of the prize, all bidders participate for sure and expect to make a positive gain. Moreover, as the monetary value goes to infinity, the highest bid in the support also goes to infinity.

The intuition behind Theorem 8 is quite intuitive, an increased probability of participation deteriorates the probability of winning the auction and, therefore, erodes the profitability of entering the auction. If too many bidders enter, then the entire expected surplus vanishes. Indeed, when this number goes to infinity, the individual probability of participating becomes negligible and the remaining probabilities over bids all converge to zero. Similar, if 
the costs of bidding become too high, these costs cannot be recovered by the expected gains of winning and bidders start to increase their probability of nonparticipating. Whenever, the probability of nonparticipation is positive, the expected NE payoffs are zero by Lemma 6. In that case, we arrive at a result similar to the classic result known for markets with endogenous entry: under a fixed market entrance fee, potential producers enter as long as there are expected net profits from doing so are larger than the entrance fee. In the symmetric NE of the auction, costly bidding erodes expected positive net gains until these expected gains are equal to the payoff of nonparticipation. The value of the prize has a different effect compared to the previous two parameters: If it becomes large enough, all bidders will participate and expect to make a profit. Moreover, a higher monetary value make higher bids more attractive and, therefore, increases the support.

This theorem is shown in three lemma's, each involves one of the three key parameters, that also provide some additional insights. First, we consider large numbers of bidders.

Lemma 9 Let $x \in \Delta^{N E}(M, c, n+1)$. For any $n+1 \geq \frac{M}{c}$, then $x_{N}>0$ and $u(x, x)=0$. If $n+1$ goes to infinity, then $x_{N}$ goes to 1 .

Proof. For arbitrary $n+1$ and $x \in \Delta^{N E}(M, c, n+1)$, define $\operatorname{Pr}(x ; n+1) \leq \frac{1}{n+1}$ as the probability that bidder 1 wins the auction. Note that the positive probability that nobody wins the auction, which is at least $x_{0}^{n+1}>0$, yields $p(x ; n+1)<\frac{1}{n+1}$. As $n+1$ goes to $\infty$, this probability converges to 0 . Suppose for all $n+1$ that $x_{N}(n+1)=0, x(n+1) \in$ $\Delta^{N E}(M, c, n+1)$. Then, the expected NE payoff is at most $p(x ; n+1) M-c<\frac{1}{n+1} M-c$. The upper bound converges to $-c$ as $n+1$ goes to $\infty$. Negative NE payoffs contradict Proposition 1. So, for large enough $n+1, x_{N}>0$. Since $p(x ; n+1)<\frac{1}{n+1}$, bidder 1 would certainly make a loss if the upper bound $\frac{1}{n+1} M-c$ becomes non-positive, which yields the lower bound on $n+1$. Finally, for all $n+1 \geq \frac{M}{c}, u(x, x)=0=M f_{0}(x)-c$ implies that $x_{0}=1-\left(\frac{c}{M}\right)^{\frac{1}{n}} \rightarrow 0$ as $n+1$ goes to $\infty$. By Lemma, $7, x_{0}$ is the largest probability in $S(x)$ and, therefore, all probabilities in the support converge to 0 . Hence, in the limit $x_{N}=1$. 
The lower bound on the number of bidders is derived from the intuition that the probability of winning the auction should yield a sufficiently large expected revenue to cover the cost of making a bid. In case all bidders enter the auction for sure, the probability of winning would converge to 0 if the number of bidders would go to infinity, which means that for too many bidders nonparticipation becomes a profitable deviation. Note that this intuition provides a sufficient condition for $x_{N}>0$ that is, however, not necessary because it is derived under overestimation of the true probability of winning by comparing it to a standard lottery. Note that the lower bound on the number of bidders can also be rewritten as $c \geq \frac{M}{n+1}$, meaning that the expected gain of a standard lottery ticket would be non-positive. Since the true probability of winning will be strictly smaller than in a lottery, there might be a set of consecutive $n+1$ just below $\frac{M}{c}$ for which $x_{N}>0$ also holds. This statement is illustrated by the numerical example of Section 3 where $x_{N}=.2887$ and $n+1<\frac{M}{c}$ in case of $n+1=3, M=4$ and $c=1$. So, for given $M$ and $c$, we may only expect symmetric $\mathrm{NE}$ with positive expected NE payoffs for a subset of sufficiently small consecutive numbers $n+1<\frac{M}{c}$. As the number of bidders approaches infinity, the probability that an individual bidder enters becomes negligible. The probability distribution over the numerical support has limit probabilities of zero. Under zero bidding costs, Östling et al. (2007) derive a uniform limit distribution over numerical bids with positive probabilities. Our result shows, however, that when bidding costs are positive, it is nonparticipation that prevails in the limit.

The second lemma puts an upper bound upon the costs for positive expected NE payoffs.

Lemma 10 For any $x(1) \in \Delta^{N E}(M, 1, n+1)$ such that $u(x(1), x(1))=f_{0}(x(1)) M-$ $1 \geq 0$ and all $c \leq f_{0}(x(1)) M$ it holds that $x(1) \in \Delta^{N E}(M, c, n+1)$. Moreover, for $c>1, x(c) \in \Delta^{N E}(M, c, n+1)$ such that $u(x(c), x(c))=f_{0}(x(c)) M-c>0$ implies that $x(c) \in \Delta^{N E}(M, 1, n+1)$. 
Proof. Consider $x \in \Delta^{N E}(M, 1, n+1)$. By Lemma 6, for all $i \in S^{N}(x)$ and $j \in B^{N}$ :

$$
(M-i) f_{i}(x)-1 \geq(M-j) f_{j}(x)-1 \Leftrightarrow(M-i) f_{i}(x)-c \geq(M-j) f_{j}(x)-c
$$

and equality holds if also $j \in S^{N}(x)$. So, different $c$ do not affect these inequalities. Then, $x$ is also a NE for $c$ provided that the expected utility under $c$, which is $f_{0}(x) M-c$, obeys the non-negativity implied by Proposition 1 . Starting with arbitrary $c \geq 1$ yields the second statement, where the condition $f_{0}(x(c)) M-c>0$ ensures that $S(x(c))=S^{N}(x(c))$ and $x_{0}(\hat{c})<1-\left(\frac{\hat{c}}{M}\right)^{\frac{1}{n}}$ for all $\hat{c}=1, \ldots, c$ by $(3)$.

This last result is based upon the observation that the equilibrium conditions for numeric bids in $B^{N}$ are invariant with respect to the costs of making a bid. So, as long as the expected payoffs of participating for sure under $x(1) \in \Delta^{N E}(M, 1, n+1)$ under different costs $c$ cover at least the opportunity costs of nonparticipation, participation by playing $x(1)$ remains a NE. This invariance cannot hold for any $\mathrm{NE} x(c) \in \Delta^{N E}(M, c, n+1)$ with a positive probability of nonparticipation, that is $x_{N}(c)>0$, because then $u(x, x)=0$ implies that $x_{0}(c)=1-\left(\frac{c}{M}\right)^{\frac{1}{n}}$ depends upon $c$. Moreover, the probability $x_{0}(c)$ is decreasing in $c$. Therefore, $x(c) \in \Delta^{N E}(M, c, n+1)$ such that $x_{N}(c)>0$ implies $x(c) \notin \Delta^{N E}\left(M, c^{\prime}, n+1\right)$ for $c^{\prime} \neq c$. A similar invariance also fails for $M$ and $M^{\prime}$. Therefore, $x \in \Delta^{N E}(M, c, n+1)$ implies $x \notin \Delta^{N E}\left(M^{\prime}, c, n+1\right)$.

The following result states that for large enough monetary values, all bidders participate for sure and expect to make a profit.

Lemma 11 Let $x(M) \in \Delta^{N E}(M, c, n+1)$. For sufficiently large $M, x_{N}(M)=0$ and $u(x(M), x(M))>0$. If $M$ goes to infinity, then $\bar{\beta}(M)$ also goes to infinity, where $\bar{\beta}(M)$ is the upper bound on the numerical support $S^{N}(x(M))$.

Proof. Suppose not, then for all $M>0$ and $x(M)$ we would have $x_{N}(M)>0$ and $u(x(M), x(M))=0$. By $(3)$, as $M$ goes to $\infty, x_{0}(M)=1-\left(\frac{c}{M}\right)^{\frac{1}{n}} \rightarrow 1$ and, therefore, 
$x_{N}(M) \rightarrow 0, x_{1}(M) \rightarrow 0$ and

$$
f_{1}(x(M))=\sum_{k_{0} \neq 1}^{n}\left(\begin{array}{c}
n \\
k_{0}
\end{array}\right) x_{0}^{k_{0}}(M)\left(1-x_{0}(M)-x_{1}(M)\right)^{n-k_{0}} \rightarrow 1 .
$$

Then, for sufficiently large $M, \hat{x}^{1}=(0,0,1,0, \ldots, 0)$ yields $u\left(\hat{x}^{1}, x(M)\right)>0$, which is a profitable deviation from the NE $x(M)$, a contradiction. Hence, for sufficiently large $M$, $x_{0}(M)<1-\left(\frac{c}{M}\right)^{\frac{1}{n}}$ and, by (3), this implies $u(x(M), x(M))>0$. Finally, we show that $\lim _{M \rightarrow \infty} \inf \bar{\beta}(M)=\infty$. Suppose not, then for the symmetric NE $x(M)$ with the smallest numerical support $S^{N}(x(M))$ we would have $\lim _{M \rightarrow \infty} \bar{\beta}(M)=\bar{\beta}<\infty$. By Lemma 6 we have $f_{i}(x(M))<f_{i+1}(x(M))$ for $i, i+1 \leq \bar{\beta}$. The arguments in this lemma's proof also apply to $i=\bar{\beta}$ and $i+1=\bar{\beta}+1$. Hence, $f_{0}(x(M))<\ldots<f_{\bar{\beta}}(x(M))<f_{\bar{\beta}+1}(x(M))$. By definition of the NE concept, $(M-\bar{\beta}) f_{\bar{\beta}}(x(M))-c \geq(M-\bar{\beta}-1) f_{\bar{\beta}+1}(x(M))-$ c. However, as $M$ goes to $\infty, f_{\bar{\beta}}(x(M)) \geq \frac{M-\bar{\beta}-1}{M-\bar{\beta}} f_{\bar{\beta}+1}(x(M)) \rightarrow f_{\bar{\beta}+1}(x(M))$, which contradicts $f_{\bar{\beta}}(x(M))<f_{\bar{\beta}+1}(x(M))$. Hence, $\lim _{M \rightarrow \infty} \inf \bar{\beta}(M)=\infty$.

Under symmetric strategies, the random number of participating bidders follows a Binomial distribution with $n+1$ draws and a probability $1-x_{N}$ of participation. Therefore, the expected number of bidders is $(n+1)\left(1-x_{N}\right)$ with a standard deviation of $\sqrt{(n+1) x_{N}\left(1-x_{N}\right)}$. Obviously, if $x_{N}=0$, then all $n+1$ bidders enter for sure. Similar, $x_{N}=1$ implies no bidder enters for sure.

A final remark concerns the case no bidding costs case $c=0$. The assumption $c>0$ is motivated by eradicating many counter intuitive asymmetric NEs, such as bidder 1 bids 0 and all others bidders bid at least 1 . In case of symmetric NEs, the above results also hold for $c=0$. Then, in any symmetric $x \in \Delta^{N E}$ we have $u(0, x)=M f_{0}(x)>0$ and, hence, $u(x, x)>0$ and $x_{N}=0$ (all bidders participate for sure).

\section{Computation of Symmetric Nash Equilibria}

The results derived in the previous section state general properties of symmetric NEs. In this section, we identify a mathematical program and sufficient conditions under which the 
optimum of this program coincides with one of these symmetric NEs. This program also can answer whether the expected NE utilities are positive or zero. As in the previous section, we assume without loss of generality that $n+1 \geq 3$.

The mathematical program is given by

$$
\begin{aligned}
& \min _{z \geq 0 ; x \geq 0} z \\
& \text { s.t. } \\
& u(i, x) \quad \leq z, \quad i \in B^{N}, \\
& \sum_{i \in B^{N}} x_{i} \leq 1,
\end{aligned}
$$

where $u(i, x)=(M-i) f_{i}(x)-c$ for $i \in B^{N}$. Since all $u(i, x)$ are independent of $x_{N}$ we have implicitly defined this probability as the slack in the last constraint.

The following results are rather straightforward. Let $x \in \Delta^{N E}$ and $z=u(x, x) \geq 0$, then the standard properties of the NE concept imply that $(z, x)$ is a feasible solution of (4). This fact implies a non-empty set of feasible solutions and that the optimal $z \geq 0$ is bounded from above by $\min _{x \in \Delta^{N E}} u(x, x)$. Furthermore, the subset of $x \geq 0$ such that $\sum_{i \in B^{N}} x_{i} \leq 1$ is a non-empty, compact and convex set and, by $(2), u(i, x), i \in B^{N}$, is a continuous function in $x$. This suffices for program (4) to admit an optimal solution.

Recall that, depending upon the parameter values, the expected NE utilities could be positive or zero. In case there exists an $x \in \Delta^{N E}$ such that $z=u(x, x)=0$, then $(0, x)$ is an optimal solution. Unfortunately, every $y \in \Delta$ such that $(0, y)$ is feasible in (4) is also an optimal solution and, therefore, not every such $(0, y)$ corresponds to a NE. For example, for $n+1=3, M=4$ and $c=1$, we obtain from Table 1 and 2 that $x \in \Delta^{N E}$ implies $u(x, x)=0$. Implementation of (4) in the optimization package $\mathrm{GAMS}^{8}$ yields the optimal solution $(z, y)$ with $z=0$ and $y=(0.0000,0.289,0.500,0.211,0.000,0.000)$, which differs from the NE $x$. Moreover, $u(1, y)=-0.244<0$ implies that $y$ fails the NE conditions.

Based upon this example, one may easily discard program (4) as being of limited interest, but we will argue that this would be the wrong conclusion. The natural question is under what conditions the optimal solution correspond to a symmetric NE. As will become clear from the mathematical proofs, there are two conditions under which the optimal solution

\footnotetext{
${ }^{8}$ For more information on GAMS, we refer to Brooke et al. (1998).
} 
$(z, x)$ of (4) implies $x \in \Delta^{N E}$ : The expected utility $z$ in the optimal solution has to be positive and a technical condition on the probabilities of winning.

The technical condition on the winning probabilities is associated with a particular redistribution of probability mass over two consecutive bids ceteris paribus keeping all other bidding probabilities fixed. Formally, for $j \in B^{N}$, consider the function $f_{j}(x)$ in case all $x$ variables on which it depends are fixed, except for $x_{i}$ and $x_{i+1}$, where $i+1 \leq j-1$. Note that by fixing all other variables, the sum $x_{i}+x_{i+1}$ is also fixed. Let $b=x_{i}+x_{i+1} \in[0,1]$ and define the one-dimensional function $g:[0, b] \rightarrow[0,1]$ as

$$
g_{i, j}(a)=f_{j}\left(x_{0}, \ldots, x_{i-1}, a, b-a, x_{i+2}, \ldots, x_{j-1}, r_{j}(x)\right) .
$$

The technical condition is as follows:

Assumption 12 For all $j \in B^{N}$ and $i+2 \leq j, g_{i, j}(a)$ is strictly quasi-convex in $a \in[0, b]$.

We postpone discussing this assumption after having established the main result of this section.

Theorem 13 Let Assumption 12 hold and $(z, x)$ is an optimal solution of (4) such that $z>0$. Then, $x \in \Delta^{N E}$ and $u(x, x)=z$ and $\sum_{i \in B^{N}} x_{i}=1$ in (4).

We prove this result in a number of lemmas. The first lemma is easily verified from (2) and stated without a proof.

Lemma 14 For $i \in B^{N}, f_{i}(x)$ is continuous on $\Delta, f_{i}(x)$ is symmetric in the variables $x_{0}, x_{1}, \ldots, x_{i-1}$.

The next lemma states some properties of the following modification of $f_{i}$ in which the variable $r_{i} \in \mathbb{R}_{+}$replaces the function $r_{i}(x)$. We call this function the modified function $f_{i}$. These properties are also easily verified from (2).

Lemma 15 The modified function $f_{i}(x)$ is non-decreasing in the variables $x_{0}, x_{1}, \ldots, x_{i-1}, r_{i}$ and, increasing if all $x_{0}, x_{1}, \ldots, x_{i-1}, r_{i}$ are positive. 
The next result relates any optimum of program (4) to the property of the support derived in Lemma 5.

Lemma 16 Let $(x, z)$ be an optimal solution of (4) such that $z>0$. Then, there exists a $\bar{\beta} \geq 0$ such that $S^{N}(x)=\{0, \ldots, \bar{\beta}\}$

Proof. Suppose not. Then, there exists an optimal solution $(x, z)$ of $(4)$ for which there exists some $i \in B^{N} \backslash\{0\}$ such that $x_{i-1}=0$ and $x_{i}>0$. The proof consist of redistributing part of the variable $x_{i}$ over $x_{0}, \ldots, x_{i-1}$ such that a new feasible solution $\left(z^{\prime}, y\right)$ is constructed with $u(j, y)<u(j, x)$ for all $j \in B^{N}$ and $0 \leq z^{\prime}<z$.

First, choose $\varepsilon_{0}, \varepsilon_{1}, \ldots, \varepsilon_{i}>0$ such that $\sum_{m=0}^{j-1} \varepsilon_{m} \leq \varepsilon_{j}$ for all $j \leq i$ and $\varepsilon_{i}<x_{i}$. Define

$$
y_{j}=\left\{\begin{array}{ll}
x_{j}+\varepsilon_{j}, & j \leq i-1, \\
x_{j}-\varepsilon_{j}, & j=i, \\
x_{j}, & j \geq i+1,
\end{array} \quad \text { and } \quad z^{\prime}=\max \{0, u(0, y), \ldots, u(M-c, y)\} .\right.
$$

Then, for all $j \in B^{N}, y_{j} \geq 0$ (with strict inequality for all $j \leq i$ ) and $\sum_{i=0}^{M-c} y_{i}=\sum_{i=0}^{M-c} x_{i}+$ $\sum_{j=0}^{i-1} \varepsilon_{j}-\varepsilon_{i} \leq 1$. Thus, $\left(y, z^{\prime}\right)$ is a feasible solution of $(4)$.

Next, we show that $z^{\prime}<z$. We partition all $j \in B^{N}$ according to $j \geq i$ and $j \leq i-1$.

1. $j \geq i$ : By $x_{i-1}=0$, it follows for every $j \geq i$ that $F_{j} \subseteq F_{i-1}$ and, thus, $f_{j}(x) \leq f_{i-1}(x)$. Moreover, from $x_{i}>0$ it follows that $f_{i-1}(x) \geq x_{i}^{n}>0$ and, thus for all $j \geq i$ :

$$
u(j, x)=(M-j) f_{j}(x)-c \leq(M-j) f_{i-1}(x)-c<(M-i+1) f_{i-1}(x)-c=u(i-1, x) .
$$

Hence, $z \geq u(i-1, x)>u(j, x)$ for $j=i, i+1, \ldots, M-c$ and, in particular, $u(i, x)<$ $z$. Since the maximal component-wise difference between $x$ and $y$ is $\varepsilon_{i}$ it follows by continuity that $u(j, y)<z$ for all $j \geq i$ if all $\varepsilon_{0}, \ldots, \varepsilon_{i}$ are small enough. To the precise specification of sufficiently small $\varepsilon_{0}, \ldots, \varepsilon_{i}$ we turn next under 2 .

2. $j \leq i-1$ : By (2), we have that the modified function $f_{j}$ only depends on the variables $x_{0}, \ldots, x_{j-1}, r_{j} \geq 0$, where $r_{j}=1-\sum_{m=0}^{j} x_{m}$. Furthermore, $x_{i}>0$ implies $\sum_{m=0}^{j} x_{m} \leq$ $1-x_{i}<1$ and, therefore, $r_{j}=r_{j}(x)>0$. By Lemma 15 , the modified function $f_{j}(x)$ 
is continuous and non-decreasing in all $j+1$ variables. Similar, $y_{0}, \ldots, y_{j-1}, r_{j}^{\prime}>0$ with $r_{j}^{\prime}=1-\sum_{m=0}^{j} y_{m}$ and, by Lemma 15 , modified function $f_{j}(y)$ is increasing in all these $j+1$ variables. Note that $y_{m}=x_{m}+\varepsilon_{m}>x_{m}$ for all $m \leq j-1$ implies $r_{j}^{\prime}<r_{j}$. Therefore, we must choose all $\varepsilon_{0}, \ldots, \varepsilon_{i}$ such that for every $j \leq i-1$ the decrease from $r_{j}$ to $r_{j}^{\prime}$ by $\varepsilon_{j}$ dominates the combined increases from $x_{m}$ to $y_{m}$ by $\varepsilon_{m}$ for all $m \leq j-1$. Since modified $f_{j}(x)$ is continuous, symmetric in $x_{0}, \ldots, x_{j-1}$ and non-decreasing $x_{0}, \ldots, x_{j-1}, r_{j}$, there exist $0<\varepsilon_{0} \ll \ldots \ll \varepsilon_{i-1} \ll \varepsilon_{i}<x_{i}$ such that $f_{j}(y)<f_{j}(x)$ for all $j \leq i-1$, where we write $a \ll b$ if $0<a<b$ and $\frac{a}{b}>0$ is arbitrarily small. Then, $u(j, y)<u(j, x)$ for all $j \in B^{N}$, including for those under 1 .

Hence, $z^{\prime}=\max _{j}\{0, u(1, y), \ldots, u(M-c, y)\}<z$ for all $j \in B^{N}$ and this contradicts that $(x, z)$ is an optimal solution of (4).

Corollary 17 Let $(x, z)$ be an optimal solution of (4) such that $z>0$. Then, $\bar{\beta} \geq 1$.

Proof. Suppose not, meaning $\bar{\beta}=0$. Then, $z>0$ and $\bar{\beta}=0$ imply $x_{0}=1, f_{0}(x)=0$ and $f_{i}(x)=1$ for all $i \geq 1$ meaning $u(0, x)=0$ and $z=u(1, x)=M-c-1$. By Proposition 3 and Lemma 5 , the support on $B^{N}$ of any $\hat{x} \in \Delta^{N E}$ such that $\hat{z}=u(\hat{x}, \hat{x})>0$ contains the subset $\{0,1\}$. Furthermore, in any symmetric NE there is a positive probability that no bidder wins the auction, i.e., $f_{i}(\hat{x})<1$ for all $i \in S(\hat{x})$. Therefore, $\hat{z}=u(1, \hat{x})<M-c-1=z$, which violates that the optimal $(z, x)$ is such that $z \in[0, \hat{z}]$. Clearly, a contradiction.

The previous result is independent of Assumption 12, but the following result also needs this assumption.

Lemma 18 Let Assumption 12 hold and $(z, x)$ is an optimal solution of (4) such that $z>0$. Then, $i \in S(x)$ implies $u(i, x)=z$.

Proof. Suppose not. Then, for the optimal solution $(x, z)$ of (4) define $i \in S(x)$ as the largest $i \leq \bar{\beta}$ for which $u(i, x)<z$. Then, $x_{i+1}, \ldots, x_{\bar{\beta}}>0$ (by Lemma 16), $u_{i+1}(x)=\ldots=$ 
$u_{\bar{\beta}}(x)=z$ and $u_{\bar{\beta}+1}(x) \leq z$, And $u_{j}(x)<z$ for all $j \geq \bar{\beta}+2$, because $x_{\bar{\beta}+1}=\ldots=x_{j-1}=0$ implies $f_{j}(x)=f_{\bar{\beta}+1}(x)$. The proof consist of redistributing part of the probability $x_{i}$ over $x_{0}, \ldots, x_{i-1}$ and $x_{i+1}$ such that a new feasible solution $\left(z^{\prime}, y\right)$ is constructed with $u(j, y)<z$ for all $j \in B^{N}$ and $0 \leq z^{\prime}<z$.

Without loss of generality, let $i<M-c$. Otherwise, $\left(z^{\prime}, y\right)$ as constructed in the proof of Lemma 16 immediately applies in case $i=M-c$ to improve upon $(z, x)$. Here, we need a different $\left(z^{\prime}, y\right)$. Choose $\varepsilon_{0}, \varepsilon_{1}, \ldots, \varepsilon_{i}, \varepsilon_{i+1}>0$ such that $\sum_{m=0}^{j-1} \varepsilon_{m} \leq \varepsilon_{j}$ for all $j \leq i, \varepsilon_{i}<x_{i}$ and $\varepsilon_{i+1}=\varepsilon_{i}-\sum_{j=0}^{i-1} \varepsilon_{j}$. Define $y$ such that

$$
y_{j}=\left\{\begin{array}{ll}
x_{j}+\varepsilon_{j}, & j \leq i-1, \\
x_{j}-\varepsilon_{j}, & j=i \\
x_{j}+\varepsilon_{j}, & j=i+1 \\
x_{j}, & j \geq i+2
\end{array} \quad \text { and } \quad z^{\prime}=\max \{0, u(0, y), \ldots, u(M-c, y)\}\right.
$$

Then, for all $j \in B^{N}, y_{j} \geq 0$ (with strict inequality for all $j \in S(x)$ and, possibly, also for $j=\bar{\beta}+1$ in case $i=\bar{\beta}$ ) and $\sum_{i=0}^{M-c} y_{i}=\sum_{i=0}^{M-c} x_{i} \leq 1$. Note that $y$ is constructed such that $r_{j}(y)=r_{j}(x)$ for all $j \geq i+1$, which is exploited below. Thus, $\left(y, z^{\prime}\right)$ is a feasible solution of (4). We consider two cases: $j \leq i+1$ and $j \geq i+2$.

1. $j \leq i+1$ : Similar as in the proof of Lemma 16, $0<\varepsilon_{0}<<\ldots<<\varepsilon_{i-1}<<\varepsilon_{i} \ll x_{i}$ and continuity ensures $f_{j}(y)<f_{j}(x)$ for all $j=0, \ldots, i-1$ and, thus, $u(j, y)<$ $u(j, x) \leq z$. For $j=i, f_{j}(y)>f_{j}(x)$, but for sufficiently small $\varepsilon$ 's we can ensure $u(i, y)<z$. Finally, for $j=i+1$ we have $r_{i+1}(y)=r_{i+1}(x)$ and the effect in $\varepsilon_{i}$ dominates $f_{i+1}(y)$ through $y_{i}=x_{i}-\varepsilon$. Hence, by continuity, $f_{i+1}(y)<f_{i+1}(x)$ and, thus, $u_{i+1}(y)<u_{i+1}(x) \leq z$.

2. $j \geq i+2$ : For these $j$, the issue is that $\varepsilon_{i}$ and $\varepsilon_{i+1}$ are of the same magnitude (in contrast to $\varepsilon_{m} \ll \varepsilon_{i}$ for all $\left.m \leq i-1\right)$ and $y_{i}=x_{i}-\varepsilon_{i}$ and $y_{i+1}=x_{i+1}+\varepsilon_{i+1}$ have opposite effects on $f_{j}(y)$. We have to make sure that the decrease from $x_{i}$ to $y_{i}$ dominates the increase from $x_{i+1}$ to $y_{i+1}$. We first establish the following claim.

Claim 1: $x_{i+1}<x_{i}$. 
The arguments in the proof of Lemma 7 apply: $x_{i} \leq x_{i+1} \Longrightarrow f_{i}(x) \geq f_{i+1}(x)$. This implies the contradiction $u(i, x)>u(i+1, x)=z$. Hence, $x_{i+1}<x_{i}$.

Next, we concentrate on $\varepsilon_{i}$ and $\varepsilon_{i+1}$ that are of the same magnitude.

Claim 2: $g_{i, j}\left(x_{i}-\varepsilon_{i}\right)<f_{j}(x)$ for all $j \geq i+2$.

Consider $g_{i, j}(a)$ and note that $i \in S(x)$ implies that $b=x_{i}+x_{i+1}>0$. By Lemma 14, $g_{i, j}(a)=g_{i, j}(b-a)$ for every $a \in[0, b]$. By definition of strictly quasi-convexity in the variable $a$, for every $a \in\left[0, \frac{b}{2}\right)$

$$
g_{i, j}\left(\frac{b}{2}\right)=g_{i, j}\left(\frac{1}{2} a+\frac{1}{2}(b-a)\right)<\max \left\{g_{i, j}(a), g_{i, j}(b-a)\right\}=g_{i, j}(a)=g_{i, j}(b-a) .
$$

Hence, $g_{i, j}$ is minimal in $a=\frac{b}{2}$ and, as $a$ gets closer to $\frac{b}{2}, g_{i, j}(a)$ strictly decreases. Since $x_{i+1}<x_{i}$, we have that $x_{i}>\frac{b}{2}$ and, thus, $g_{i, j}\left(x_{i}-\varepsilon_{i}\right)<g_{i, j}\left(x_{i}\right)=f_{j}(x)$ for $x_{i}-\varepsilon_{i}>\frac{b}{2}$.

Claim 3: $f_{j}(y)<f_{j}(x)$.

Define $y^{\prime} \in \Delta$ by $y_{i}^{\prime}=x_{i}-\varepsilon_{i}, y_{i+1}^{\prime}=x_{i+1}+\varepsilon_{i}$ and $y_{l}^{\prime}=x_{l}$ if $l \neq i, i+1$. Then, the length (norm) $\left|y-y^{\prime}\right|$ is arbitrary small compared to $|x-y|$. Thus, by continuity of the function $f_{j}$ we have that $f_{j}\left(y^{\prime}\right)=g_{i, j}\left(x_{i}-\varepsilon_{i}\right)<f_{j}(x)$ implies that $f_{j}(y)<f_{j}(x)$. Claim 3 implies that $u_{j}(y)<u_{j}(x)$ for $j=i+2, i+3, \ldots, M-c$.

Hence, $u(j, y)<z$ for all $j \in B^{N}$ and, thus, $z^{\prime}<z$ which contradicts that $(x, z)$ is an optimal solution of (4).

The following lemma completes the proof of Theorem 8.

Lemma 19 Let Assumption 12 hold and $(z, x)$ is an optimal solution of (4) such that $z>0$. Then, $x \in \Delta$ is a symmetric $N E, u(x, x)=z$, and $\sum_{i \in B^{N}} x_{i}=1$ in (4).

Proof. By Lemma 18, for every $i \in S(x): u(i, x)=z$. Hence, $u(x, x)=z$. Next, for every $j \in B^{N}: u(j, x) \leq z$ and, thus, for every $y \in \Delta: u(y, x) \leq z$. Hence, $x$ is a symmetric 
NE such that $u(x, x)=z$. Finally, we show that $x_{N}=0$. Suppose not, then $(z, x)$ is such that $x_{N}=1-\sum_{i \in B^{N}} x_{i}>0$. For $0<\varepsilon<x_{N}$, define $y \in \Delta$ such that $y_{N}=x_{N}-\varepsilon$, $y_{\bar{\beta}}=x_{\bar{\beta}}+\varepsilon$ and $y_{i}=x_{i}$ for all other $i \neq \bar{\beta}$. Then, for sufficiently small $\varepsilon, u(i, y)<z$ for all $i \geq \bar{\beta}$. Since also $u(i, y)=u(i, x)=z$ for all $i \leq \bar{\beta}-1$, we have that $(z, y)$ is another optimal solution in (4). Since $y$ fails the property of Lemma 18, we arrived at a contradiction. Hence, $x_{N}=0$ implies $\sum_{i \in S^{N}(x)} x_{i}=\sum_{i \in B^{N}} x_{i}=1$ in $(4)$.

Note that the proof of Theorem 8 consists of two crucial steps: Lemma 16 and 18. The proof of Lemma 16 is independent of Assumption 12, but does require $z>0$, because otherwise the arguments would fail. Similar, $z>0$ is necessary in the proof of Lemma 18 and, additionally, Assumption 12 is a sufficient condition. Under this assumption, the redistribution of probabilities described by $y$ in (6) improves the objective function of the program (4).

It is unclear whether Assumption 12 covers the entire class of auctions considered in this paper. By the symmetry of Lemma 15, we may without loss of generality take $i=0$ in verifying this assumption for all $M$ and $c$. Verification whether $g_{i, j}(a)$ is strictly quasiconvex for all $i, j \geq i+2$, all $n+1 \geq 3$ and all $x \in \Delta$ is a Herculean task. Assumption 12 is less restrictive than assuming $f_{j}(x)$ is strictly (quasi-)convex in all $x_{0}, \ldots, x_{j-1}$. Note that strictly convex can be shown for $n+1=3,4$, all $a \in[0,1]$ and all other $x_{m} \in \mathbb{R}_{+}$, which follows directly from $g_{i, j}^{\prime \prime}(a)>0 .{ }^{9}$ So, Assumption 12 does specify a non-empty subclass of auctions. The latter approach, however, is too crude and breaks down for $n+1 \geq 5$, because then odd powers of the third degree or higher with negative coefficients might appear in $g_{i, j}^{\prime \prime}(a)$. Introducing the constraints $a \leq b=x_{0}+x_{1}$ and $x \in \Delta$ make verification of the sign of $g_{i, j}^{\prime \prime}(a)$ unworkable for arbitrary parameter values, because the function $g_{i, j}(a)$ is highly nonlinear and the length of the support is unknown. For the same reasons, application of the necessary and sufficient conditions for additively decomposed quasi-convex function in Crouzeix and Lindberg (1986) to (5) is also unworkable.

\footnotetext{
${ }^{9}$ Upon request, a proof is available from the authors.
} 
The optimal strategy of program (4) can also be interpreted as the maximin value of a game where a single bidder faces a bidding ring formed by all other bidders that are restricted to all follow the same bidding strategy. Then, the larger the single bidder's expected utility, the lower the ring's expected utility is, which suggests a competitive game. So, by minimizing the single bidder's expected utility the ring gives away the least expected amount. By symmetry of all bidders in the auction, the ring's minimizing bidding strategy is also one of the single bidder's best responses to this minimizing bidding strategy. Therefore, if $(z, x)$ solves (4) such that $z>0$, then $(x, x) \in \arg \max _{x^{\prime} \in \Delta} \min _{x^{\prime \prime} \in \Delta} u\left(x^{\prime}, x^{\prime \prime}\right)$. By von Neumann's famous maximin equals minimax theorem, we also have that $(x, x) \in \arg \min _{x^{\prime \prime} \in \Delta} \max _{x^{\prime} \in \Delta} u\left(x^{\prime}, x^{\prime \prime}\right)$.

The results obtained thus far have several implications (under Assumption 12):

1. If all symmetric NE have positive expected NE utilities, then (4) identifies the symmetric NE with the lowest expected NE utilities and, consequently, $z>0$.

2. If $(z, x)$ is an optimal solution and $z>0$, then this rules out any symmetric NE with expected $\mathrm{NE}$ utilities equal to 0.

3. If $(z, x)$ is an optimal solution and $z=0$, then there exists a symmetric NE with expected NE utilities of 0 (because the if of point 1. cannot hold).

These implications make program (4) very interesting for numerical analyses, because this program can answer the question whether for certain parameter values the expected $\mathrm{NE}$ utilities are positive or zero and, if positive, it yields a symmetric NE.

\section{Numerical Analysis of Symmetric NEs}

As has become clear from the previous sections, the functional form of the winning probabilities in (2) is the complicating factor in fully characterizing the symmetric NE of the unique-lowest-bid auction. To obtain additional insights, we resort to three numerical methods. First, we further explore GAMBIT. Second, we implement the optimization program (4) 
in the numerical optimization package GAMS. Third, simulation of the replicator dynamics in Maple ${ }^{10}$ to touch upon an issue neglected thus far: The evolution of bidding behavior.

In GAMBIT, we computed all NE and implemented a sufficiently large subset of $B$. This slowed down the computations considerably in computing NEs when $n+1$ and $M$ increase. Since also the implementation in GAMBIT is time consuming, we restricted the use of this package to $n+1=3$ or 4 , and parameter values $M$ in the range of 3 to 25 and $c=1$. GAMBIT always reported a single symmetric NE. A fast and efficient way to compute the symmetric NE under GAMBIT is to compute the quantal response equilibrium, see McKelvey and Palfrey (1995). Since the quantal response equilibrium may fail to be evolutionary stable, we cannot conclude evolutionary stability from this equilibrium concept.

Program (4) is implemented in GAMS and, for all parameter values run, GAMS reports normal completion. For $n+1 \geq 8$, our computer program becomes very slow due to the need to compute a large amount of multinomial coefficients prior to the numerical optimization. For that reason, we did not perform any computations in GAMS for $n+1 \geq 10$.

Next, we briefly introduce the replicator dynamics. Bidders in the lowest-unique-bid auction come from a single population and any vector in $\Delta$ represents the population fractions of bids in this population. Time $t \in \mathbb{R}_{+}$is continuous and $x(t) \in \Delta$ represents the population fractions at time $t$, where $x(0)$ represents the initial population fractions. We assume that the population size $N \gg n+1$ such that modifications due to finite populations can be neglected. The replicator dynamics are defined as in e.g. Weibull (1995) and given by the following system of differential equations:

$$
\dot{x}_{b}(t)=x_{b}(t)[u(b, x(t))-u(x(t), x(t))], \quad b \in B .
$$

In Bukowski and Miekisz (2004), it is shown that the set of evolutionary stable strategies (ESS) is a subset of the set of asymptotically stable equilibrium (ASE) strategies and that the latter is a subset of the set of NE. Formally, $\Delta^{E S S} \subseteq \Delta^{A S E} \subseteq \Delta^{N E}$. Verification of the definition of ESS for the lowest-unique-bid auction or of the optimum of program (4) is too

\footnotetext{
${ }^{10}$ For more information on Maple we refer to www.maplesoft.com.
} 
difficult given that we cannot fully characterize NEs or this optimum. The quantal response equilibrium may not be ESS, see McKelvey and Palfrey (1995). For that reason, we resort to numerically solving for stationary distributions of (7). In case of convergence, numerical methods for simulating time paths of replicator dynamics (7) yield ASE strategies and this is as close as we (numerically) can get to ESS. All numerical simulations were performed in the software package Maple by invoking the fourth-order Runga-Kutta method with a step size of 0.01, see e.g., Betounes (2001). The initial population fractions were chosen as the uniform distribution over a large support of consecutive numbers containing 0 . In all simulations, the initial support $S(x(0)) \supset S\left(x^{*}\right)$, where $x^{*}=\lim _{t \rightarrow \infty} x(t)$. The replicator dynamics converged in all parameter values run, but convergence slows down considerably for $n+1 \geq 8$.

For $n+1=3$ or 4 , we first computed all NEs with Gambit for several parameter values $3 \leq M \leq 25$ and $c=1$. Then, we ran the replicator dynamics and obtained convergence to the symmetric NE. In case $x \in \Delta^{N E}$ such that $u(x, x)>0$, we also performed the numerical optimization algorithms in GAMS. This was done to check the numerical accuracy of the limit solution of the replicator dynamics. Since GAMS returned approximately $\left(u\left(x^{*}, x^{*}\right), x^{*}\right)$, all limit solutions $x^{*}$ of the replicator dynamics are very accurate and $\left(u\left(x^{*}, x^{*}\right), x^{*}\right)$ is feasible in (4). In all these cases, all three numerical methods provide consistent and reinforcing results.

Table 4 reports several numerical solutions obtained from both (4) and the replicator dynamics (7), where .000 indicates a positive probability that is insignificant. Blank spaces indicate a solution with at least 7 digits equal to 0 that, therefore, we presume are zero.

Roughly speaking, the support of the symmetric NE grows if either $M$ increases or the number of bidders $n+1$ increases, where $c$ is fixed. More bidders increase the competition for the single item for sale and bidders spread out their bids in order to seek profitable opportunities. However, for small numbers of bidders, roughly the range $n+1 \leq 6$, Table 4 shows non-monotonic behavior with respect to the size of the support. For $n+1=3$, this 


\begin{tabular}{|c|c|c|c|c|c|c|c|c|}
\hline$n+1$ & $M$ & $x_{0}$ & $x_{1}$ & $x_{2}$ & $x_{3}$ & $x_{4}$ & $x_{5}$ & $x_{6}$ \\
\hline \multirow[t]{4}{*}{3} & 50 & 0.464 & 0.258 & 0.148 & 0.091 & 0.040 & & \\
\hline & 100 & 0.460 & 0.253 & 0.142 & 0.083 & 0.057 & 0.005 & \\
\hline & 200 & 0.458 & 0.251 & 0.139 & 0.079 & 0.047 & 0.026 & \\
\hline & 400 & 0.457 & 0.250 & 0.137 & 0.076 & 0.044 & 0.028 & 0.007 \\
\hline \multirow[t]{4}{*}{4} & 50 & 0.452 & 0.428 & 0.119 & & & & \\
\hline & 100 & 0.450 & 0.427 & 0.122 & 0.000 & & & \\
\hline & 200 & 0.449 & 0.426 & 0.124 & 0.001 & & & \\
\hline & 400 & 0.448 & 0.425 & 0.125 & 0.001 & & & \\
\hline \multirow[t]{4}{*}{5} & 50 & 0.364 & 0.320 & 0.193 & 0.095 & 0.028 & & \\
\hline & 100 & 0.361 & 0.318 & 0.192 & 0.096 & 0.033 & & \\
\hline & 200 & 0.359 & 0.316 & 0.192 & 0.095 & 0.034 & 0.003 & \\
\hline & 400 & 0.359 & 0.316 & 0.191 & 0.095 & 0.035 & 0.004 & \\
\hline \multirow[t]{4}{*}{6} & 50 & 0.331 & 0.301 & 0.234 & 0.118 & 0.016 & & \\
\hline & 100 & 0.329 & 0.299 & 0.233 & 0.120 & 0.019 & & \\
\hline & 200 & 0.328 & 0.298 & 0.232 & 0.122 & 0.020 & & \\
\hline & 400 & 0.327 & 0.298 & 0.232 & 0.122 & 0.021 & 0.000 & \\
\hline \multirow[t]{4}{*}{7} & 50 & 0.299 & 0.274 & 0.228 & 0.140 & 0.054 & 0.005 & \\
\hline & 100 & 0.297 & 0.272 & 0.226 & 0.140 & 0.056 & 0.008 & \\
\hline & 200 & 0.296 & 0.271 & 0.226 & 0.141 & 0.057 & 0.010 & \\
\hline & 400 & 0.295 & 0.271 & 0.225 & 0.141 & 0.058 & 0.010 & \\
\hline \multirow[t]{4}{*}{8} & 50 & 0.275 & 0.255 & 0.220 & 0.157 & 0.078 & 0.014 & \\
\hline & 100 & 0.273 & 0.253 & 0.219 & 0.157 & 0.080 & 0.017 & \\
\hline & 200 & 0.272 & 0.252 & 0.218 & 0.157 & 0.081 & 0.019 & 0.000 \\
\hline & 400 & 0.272 & 0.252 & 0.218 & 0.157 & 0.082 & 0.019 & 0.001 \\
\hline \multirow[t]{4}{*}{9} & 50 & 0.256 & 0.238 & 0.211 & 0.165 & 0.098 & 0.031 & 0.000 \\
\hline & 100 & 0.254 & 0.237 & 0.210 & 0.165 & 0.099 & 0.034 & 0.003 \\
\hline & 200 & 0.253 & 0.236 & 0.209 & 0.164 & 0.100 & 0.035 & 0.004 \\
\hline & 400 & 0.252 & 0.235 & 0.209 & 0.164 & 0.100 & 0.036 & 0.004 \\
\hline
\end{tabular}

Table 4: Symmetric NEs for several values of $M, n+1$ and $c=1$.

can be explained by the fact that the highest bid wins if the other two bidders make the same (lower) bid. In case of $n+1=4$, the highest bid can only win if the other three bidders all make the same bid, which is less likely then two bidders making the same bid. So, the higher bids are less attractive and the support retracts. For $n+1=5$, the probability of winning with the highest bid is somewhat better again, because it also wins for example if two opponents both bid some lower bid and the other two opponents both bid some other lower bid. When $n+1$ increases further, these kind of odd/even considerations loose significance 


\begin{tabular}{|c|c|c|c|c|c|c|c|c|c|c|}
\hline$n+1$ & M & $x_{N}$ & $x_{0}$ & $x_{1}$ & $x_{2}$ & $x_{3}$ & $x_{4}$ & $x_{5}$ & $x_{6}$ & $x_{7}$ \\
\hline \multirow[t]{5}{*}{4} & 1000 & & 0.4480 & 0.4251 & 0.1253 & 0.0016 & & & & \\
\hline & 10000 & & 0.4478 & 0.4249 & 0.1256 & 0.0017 & & & & \\
\hline & 100000 & & 0.4477 & 0.4249 & 0.1257 & 0.0017 & & & & \\
\hline & 1000000 & & 0.4477 & 0.4249 & 0.1257 & 0.0017 & & & & \\
\hline & 10000000 & & 0.4477 & 0.4249 & 0.1257 & 0.0017 & & & & \\
\hline \multirow[t]{5}{*}{6} & 1000 & & 0.3268 & 0.2977 & 0.2315 & 0.1223 & 0.0214 & 0.0003 & & \\
\hline & 10000 & & 0.3266 & 0.2975 & 0.2314 & 0.1225 & 0.0216 & 0.0004 & & \\
\hline & 100000 & & 0.3266 & 0.2975 & 0.2314 & 0.1225 & 0.0216 & 0.0005 & & \\
\hline & 1000000 & & 0.3266 & 0.2975 & 0.2314 & 0.1225 & 0.0216 & 0.0005 & & \\
\hline & 10000000 & & 0.3266 & 0.2975 & 0.2314 & 0.1225 & 0.0216 & 0.0005 & & \\
\hline \multirow[t]{5}{*}{8} & 1000 & & 0.2712 & 0.2514 & 0.2178 & 0.1571 & 0.0821 & 0.0196 & 0.0008 & \\
\hline & 10000 & & 0.2710 & 0.2512 & 0.2176 & 0.1571 & 0.0822 & 0.0199 & 0.0010 & \\
\hline & 100000 & & 0.2712 & 0.2512 & 0.2176 & 0.1571 & 0.0822 & 0.0199 & 0.0010 & \\
\hline & 1000000 & & 0.2712 & 0.2512 & 0.2176 & 0.1571 & 0.0822 & 0.0199 & 0.0010 & 0.0000 \\
\hline & 10000000 & & 0.2712 & 0.2512 & 0.2176 & 0.1571 & 0.0822 & 0.0199 & 0.0010 & 0.0000 \\
\hline
\end{tabular}

Table 5: Symmetric NEs for several values of $M, n+1$ and $c=1$.

\begin{tabular}{|c|cccc|}
\hline$\frac{M}{c}$ & \multicolumn{4}{|c|}{$n+1$} \\
& 3 & 4 & 5 & 6 \\
\hline \hline 3 & 0 & 0 & 0 & 0 \\
4 & 0 & 0 & 0 & 0 \\
5 & + & 0 & 0 & 0 \\
6 & + & 0 & 0 & 0 \\
7 & + & 0 & 0 & 0 \\
8 & + & + & + & 0 \\
9 & + & + & + & + \\
\hline
\end{tabular}

Table 6: Parameter values for which the expected NE utilities are either positive $(+)$ or zero (0) under $c=1$.

and, then, it seems that the numerical support is non-decreasing in $n+1$.

Table 5 represents simulation results for increasingly large values of the prize $M$ for $n+1$ equal to 4, 6 and 8. The change in probabilities and the growth of the support is very slow. For $n+1=4$, this table might give the false impression that there is convergence to a finite support, but according to Theorem 8 this is not the case.

Table 6 reports parameter values with zero and positive expected utilities. As $n+1$ increases, the consecutive set of low values $M$ for which the expected NE utilities are 0 
grows. If the sufficient condition $n+1 \geq \frac{M}{c}$ in Theorem 8 for zero profits would also be a necessary condition, then the entire lower triangular matrix would be filled with +'s. Since it is not, the sufficient condition is only a rough indication for the parameter values for which the expected NE utilities are zero.

To summarize, GAMBIT returns a single symmetric NE. Furthermore, the replicator dynamics always converge to a symmetric NE and, if it has positive NE utilities, this limit solution is one-to-one related to the optimal solution of program (4). This gives numerical support for the following conjecture.

Conjecture 20 The lowest-unique-bid auction has a unique symmetric NE that is also ASE. If $x \in \Delta^{N E}$ and $u(x, x)>0$, then $(u(x, x), x)$ is an optimal solution of (4).

One final remark is in place. The replicator dynamics always converges, in particular if $u(x, x)=0$. Under $u(x, x)$, computing limit solutions in Maple takes considerably more time than optimization in GAMS. Therefore, we regard simulating (7) and program (4) as two complementary methods in computing the symmetric NE.

\section{Concluding Remarks}

The lowest-unique-bid auction is a very complicated auction to analyze. Nevertheless, several interesting insights are obtained for symmetric NEs such as costly endogenous entry, comparative statics, the maximin interpretation that allows for a mathematical program and the issue of evolutionary stability.

In Eichberger and Vinogradov (2008), Östling et al. (2007) and Rapoport et al. (2007) the testing of either field or laboratory experiments is the motivating issue. In the last two references, the no bidding costs assumption implies that it is always profitable to participate and endogenous entry is trivial. In contrast, our results indicate that the expected profits are zero in case the number of bidders is above a particular threshold. This threshold can be rewritten as a condition stating that the costs of purchasing a ticket in a standard singleprize lottery is at least the expected monetary gain from such a ticket if all bidders present 
have equal chance. We know from lotteries that people buy lottery tickets even though they face an expected loss. In the symmetric NE, however, bidders will avoid expected losses. This issue is currently lacking in testing laboratory experiments under zero bidding costs.

Negative expected losses might also be the reason why the unique-lowest-bid auction that featured in the daily Dutch TV show Shop4Nop was terminated in 2006. The monetary values of the daily prizes were below 1000 euro and a bid could be made by SMS at the costs of 70 euro cents, see e.g. Veldhuizen (2006). By Theorem 8, there are no positive expected gains if $n+1 \geq 1,429$ and if more participants actually enter expected losses are for sure. This number is very low for a nation wide TV show in a country with 16 million inhabitants. It seems that the audience eventually learned to play this game and found out that there is little fun in placing bids in an auction that theoretically has no positive expected gains. This might explain why the show's popularity dropped over time and new activity rules to attract more bids were introduced after more than a year. Eventually, the show was canceled because of its low profitable for the biggest commercial TV station in The Netherlands. This anecdotal evidence suggests that the life cycle of lowest-unique-bid auctions as a commercial activity will be short. Only if the organizer is willing to accept substantial losses as part of a marketing campaign, then it might be fun playing this auction format. Whether it is a more effective marketing campaign than a standard lottery remains to be seen.

\section{References}

Becker, J. and D. Damianov (2006). On the existence of symmetric mixed strategy equilibria. Economics Letters 90, 84-87.

Betounes, D. (2001). Differential Equations: Theory and Applications with Maple. Berlin: Springer Verlag.

Brooke, A., D. Kendrick, A. Meeraus, and R. Raman (1998). Gams: A users guide. available at the GAMS Development Corporation web site at http://www.gams.com. 
Bukowski, M. and J. Miekisz (2004). Evolutionary and asymptotic stability in symmetric multi-player games. International Journal of Game Theory 33, 41-54.

Crouzeix, J.-P. and P. Lindberg (1986). Additively decomposed quasiconvex functions. Mathematical Programming 35, 42-57.

Eichberger, J. and D. Vinogradov (2008). Least unmatched price auctions. mimeo, University of Heidelberg.

Krishna, V. (2002). Auctiontheory. San Diego, CA: Academic Press.

McKelvey, R. and T. Palfrey (1995). Quantal response equilibria for normal form games. Games and Economic Behavior 10, 6-38.

McKelvey, R., D. Richard, A. McLennan, and T. Turocy (2006). Gambit: Software tools for game theory, version 0.2006.01.20. http://econweb.tamu.edu/gambit.

Myerson, R. (1998). Population uncertainty and poisson games. International Journal of Game Theory 27, 375-392.

Myerson, R. (2000). Large poisson games. Journal of Economic Theory 94, 7-45.

Östling, R., J. Wang, E. Chou, and C. Camerer (2007). Field and lab convergence in poisson lupi games. SSE/EFI working paper series in Economics and Finance 671.

Rapoport, A., H. Otsubo, B. Kim, and W. Stein (2007). Unique bid auctions: Equilibrium solutions and experimental evidence. discussion paper, University of Arizona.

Raviv, Y. and G. Virag (2007). Gambling by auctions. mimeo.

Veldhuizen, D. (2006). Shop4nop: Een veiling winnen zonder te hoeven betalen. Bachelor thesis, VU University Amsterdam, Amsterdam.

Weibull, J. (1995). Evolutionary game theory. Cambridge: MIT Press. 